\title{
Controller design for partial decoupling of linear multivariable systems
}

\author{
S.R. Weller \\ Dept. of Electrical and Electronic Engineering, \\ University of Melbourne, \\ Parkville, Vic. 3052, \\ Australia \\ e-mail: s.weller mullian.ee.mu.oz.au \\ Telephone: +61 33446299 Fax: +61 33446678 \\ G.C. Goodwin \\ Centre for Industrial Control Science, \\ Dept. of Electrical and Computer Engineering, \\ University of Newcastle, \\ University Drive, Callaghan, N.S.W., 2308 \\ Australia \\ March 1994 \\ First revision January 1995
}

Technical Report No. EE9313

\begin{abstract}
In the design of feedback control systems for linear multivariable plants, insisting on the elimination of coupling in closed-loop is often achieved at the expense of an increase in the multiplicity of infinite and non-minimum phase zeros beyond those of the plant. This behaviour is known as the "cost of decoupling" since these additional zeros are manifested in the time domain by increased rise times and undershoots in step responses. Using partially decoupling controllers, however, it is always possible to obtain closed-loop
\end{abstract}


systems with precisely the same number of infinite and nonminimum phase zeros as the plant, albeit at the expense of a restricted form of transient coupling. This paper uses a generalization of the interactor matrix to generate a class of partially decoupling controllers for square, stable plants in which diagonal decoupling arises as a special case, thereby permitting the designer to trade off speed of response versus the severity of transient interaction. 


\section{Introduction}

In the theory of linear multivariable systems, the characterization of all stabilizing controllers for a given plant was first considered by Youla, Jabr and Bongiorno (1976), and subsequently extended and refined by many authors; see e.g. Desoer, Liu, Murray and Saeks (1980), Zames (1981) and Pernebo (1981). This work is of fundamental significance in control system design, since it identifies those characteristics of a plant which impose limits on performance given the constraints of linearity, time-invariance and causality.

A natural extension of this work arises when the closed-loop transfer matrix is constrained to be diagonal; that is, the closed-loop is diagonally decoupled. It is known that the closed-loop transfer matrix obtained by a proper stabilizing compensator necessarily inherits the non-minimum phase characteristics and zero structure at infinity of the plant (Callier and Desoer 1982). Desoer and Gündeş (1986) have used algebraic arguments to show that, for a wide class of lumped and distributed continuous-time and discrete-time plants, constraining the closed-loop transfer matrix to be diagonal may introduce these zeros with greater multiplicity than the corresponding zeros in the plant. Since non-minimum phase zeros are typically associated with performance penalties - increased rise-times and undershoot in step responses - this behaviour is known as the "cost of decoupling". Moreover for ill-conditioned plants, decoupling controllers have been shown to be potentially very sensitive to uncertainty on the plant inputs (Skogestad, Morari and Doyle 1988).

An alternative design goal is that of partial (also one-way or triangular) decoupling (see, e.g. Morari and Zafiriou (1989), Stephanopoulos (1984) and Hung and Anderson (1979)) in which the closed-loop transfer matrix is lower triangular. Thus the first $i-1$ components of the plant output are unaffected by variations in the $i$ th and subsequent components of the reference input. Partially decoupled designs are generally much less sensitive to input uncertainty than diagonally decoupled designs, albeit at the expense of a restricted form of interaction in the closed-loop response. Aiming for this type of restricted closed-loop interaction provides a mechanism for ranking the components of the plant output vector according to their relative importance, i.e. a given plant output experiences a (transient) disturbance only as part of the closed-loop response to a change in set-point corresponding to a relatively more important output.

A basic design goal provided for by virtually all current design methodologies (by incorporating integral action in the controller) is that of static decoupling. That is, each plant output should be capable of tracking step changes in the corresponding reference input with asymptotically zero error, 
and the control action taken should introduce at worst only transient effects into other outputs. Since this implies diagonal decoupling at zero frequency, it suggests that for a range of low frequency reference inputs, most controllers provide for approximate decoupling. Given the discussion of diagonal and partial decoupling above, this raises the following questions: Is it possible to trade off the severity of coupling with the speed of response to reference inputs? If so, what is the nature of the design freedom exercised over this tradeoff?

These observations provide the motivation for this paper, in which a control design technique is developed which:

- highlights the implications for diagonal and partial decoupling implicit in the observation that the closed-loop transfer matrix obtained by a proper stabilizing controller necessarily inherits the plant zero structure at infinity and non-minimum phase zeros;

- provides for a family of partially decoupled designs in which diagonal decoupling arises as a special case;

- explicitly provides design freedom over a tradeoff between the severity of coupling and the speed of response.

In closely related work, Vardulakis and Karcanias (1982) define the left structure matrix in $\bar{\Omega}$ of the transfer matrix $P(s)$ as a representation of the poles and zeros of $P(s)$ lying in $\bar{\Omega}$, an "undesirable" subset of the complex plane together with the point at infinity. This work has much in common with that of Pernebo (1981a, 1981b), who introduced the concept of left and right structure matrices for multivariable systems, and gives interpretations of these matrices in terms of how well the plant output $y$ can be controlled from input $u$, and how well an unmeasured disturbance $e$ can be estimated from a vector $z$ of measured variables. Desoer and Gündeş (1986) have presented an algebraic theory for decoupling using two-degree-of-freedom controllers in which the "cost of decoupling" is made explicit. Tsiligiannis and Svoronos $(1988,1989)$ have generalized the interactor of Wolovich and Falb (1976) to extract non-minimum phase zeros from discrete-time transfer function models and have used this generalized interactor to characterize classes of designs which are optimal in a certain sense. Zafiriou and Morari (1987) have investigated the limitations imposed by unstable zeros and time delays for discrete-time multivariable systems and have formulated a design procedure which permits a quantitative evaluation of the tradeoffs between different closed-loop interaction structures. In a stochastic setting, Dugard, Goodwin 
and Xianya (1984) have shown that when designing minimum variance controllers, the variance of the tracking errors is dependent on the ordering of the outputs when the interactor is nondiagonal.

By restricting attention to plants which are open-loop stable, the developments of this paper are considerably simplified. One method of dealing with open-loop unstable plants within the proposed design framework is to pre-stabilise the plant by preliminary feedback, then to apply the proposed design method to the pre-stabilised plant. Callier and Desoer (1982) have shown that this entails no loss of design freedom in the sense that the class of closed-loop transfer matrices capable of being obtained by this method is precisely that following from the Youla parameterisation. An alternative (two-degree of freedom) method for dealing with open-loop unstable plants proposed by Weller (1993) (see also Weller and Goodwin (1995)), uses a judicious re-arrangement of the Youla parameterisation to provide important insight into how the choice of prestabilizing bandwidth impinges on the overall design.

The current paper is organized as follows: Section 2 formulates the design problem. In Section 3 the interactor of Wolovich and Falb is reviewed and it is shown how the interactor may be used to design diagonally and partially decoupled closed-loop transfer matrices for minimum phase multivariable plants. In Section 4 these results are extended to encompass nonminimum phase plants. A unified treatment of diagonal and partial decoupling is presented in Section 5 and a design example is presented in Section 6 . Conclusions are presented in Section 7.

\section{Notation}

Let $\mathbf{R}$ denote the field of real numbers, $\mathbf{C}$ the field of complex numbers, $\mathbf{R}[s]$ the ring of polynomials with coefficients in $\mathbf{R}$, and $\mathbf{R}(s)$ the field of rational functions $p(s)=n(s) / d(s)$, where $n(s), d(s) \in \mathbf{R}[s]$, and $d(s) \not \equiv 0$. A rational function $p(s)=n(s) / d(s)$ is said to be proper (resp. strictly proper) if $\partial(d(s)) \geq \partial(n(s))$ (resp. $\partial(d(s))>\partial(n(s)))$ where $\partial(n(s))$ denotes the polynomial degree of $n(s)$. The relative degree of a rational function $p(s)=n(s) / d(s)$ is defined as $\partial(d(s))-\partial(n(s))$.

Denote by $\mathbf{R}_{\mathrm{p}}(s)$ the field of proper rational functions and $\mathbf{R}_{\mathrm{p}}^{p \times m}(s)$ the set of $p \times m$ matrices whose entries are elements of $\mathbf{R}_{\mathrm{p}}(s)$. A proper rational matrix $P(s) \in \mathbf{R}_{\mathrm{p}}^{p \times m}(s)$ is said to be strictly proper (denoted $P(s) \in \mathbf{R}_{\mathrm{sp}}^{p \times m}(s)$ ) if $\lim _{s \rightarrow \infty} P(s)$ is the zero matrix in $\mathbf{R}^{p \times m}$. The units of the ring $\mathbf{R}_{\mathrm{p}}^{m \times m}(s)$ (i.e. the invertible elements) are called biproper (or bicausal) matrices, and are characterized by the property that $B(s)$ is a biproper matrix if and only 


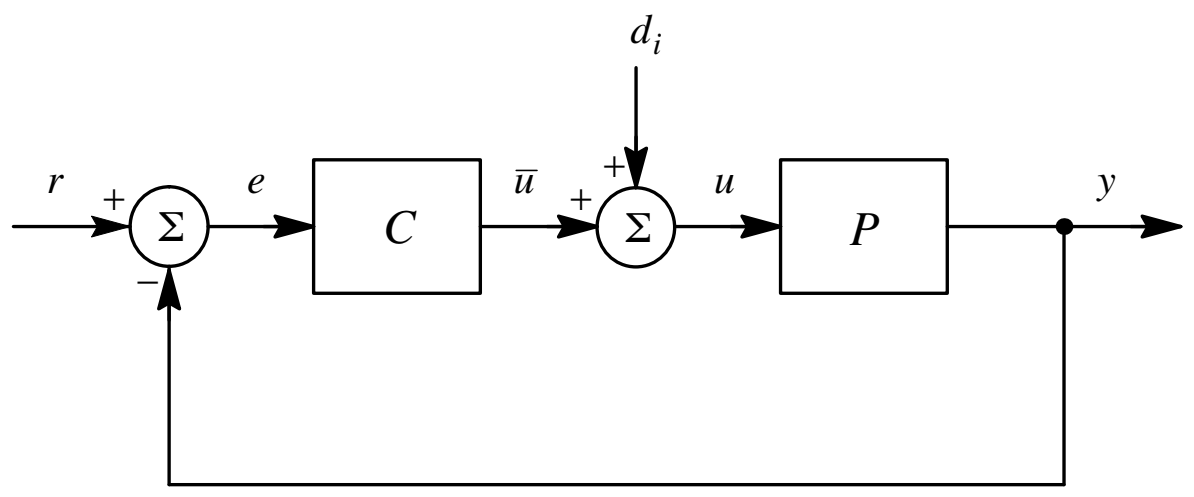

Figure 1: Feedback system $S(P, C)$ under consideration

if

$$
\operatorname{det}\left(\lim _{s \rightarrow \infty} B(s)\right) \neq 0 .
$$

Denote by $\mathbf{R}_{\mathrm{ps}}(s)$ the set of proper stable rational functions, and by $\mathbf{R}_{\mathrm{ps}}(s)$ the set of $p \times m$ matrices with entries in $\mathbf{R}_{\mathrm{ps}}(s)$. The units of $\mathbf{R}_{\mathrm{ps}}^{m \times m}(s)$ are called biproper-bistable matrices, and are characterized by the property that $B(s)$ is biproper-bistable if and only if $\operatorname{det} B(s)$ is a unit of $\mathbf{R}_{\mathrm{ps}}(s)$.

For $P(s) \in \mathbf{R}^{p \times m}(s), \mathcal{Z}[P]$ and $\mathcal{P}[P]$ denote respectively the set of transmission zeros and poles of $P$, defined via the Smith-McMillan form of $P(s)$ (Callier and Desoer 1982). In this paper, the term "zero" shall be used instead of "transmission zero", the distinction between such zeros and zeros at infinity being clear from context. A matrix is said to be unit lower triangular if it is lower triangular with 1's along the main diagonal.

\section{Problem statement}

In this paper we shall consider the multivariable feedback system $S(P, C)$ shown in Figure 1, where $P$ is the plant, $C$ is the controller to be designed, and $r, d_{\mathrm{i}}, e, \bar{u}, u$ and $y$ are, respectively, the external reference input, input disturbance, tracking error, controller output, plant input and plant output.

Throughout this paper we shall make the following assumptions regarding the plant $P$ and controller $C$ :

A1) $P$ is square, stable, nonsingular and strictly proper; 
A2) the plant $P$ and the controller $C$ have no unstable hidden modes; and

A3) $P$ has no zeros on the $j \omega$-axis.

By nonsingularity of $P$ is meant $\operatorname{det} P(s) \not \equiv 0$, i.e. there are no trivial inputs or outputs. In view of assumptions A2) and A3), $P$ has no poles on the $j \omega$-axis and, in particular, none at the origin.

The closed-loop transfer matrix of $S(P, C)$ is given by

$$
\begin{aligned}
H & =\left[\begin{array}{ll}
H_{\bar{u} r} & H_{\bar{u} d_{\mathrm{i}}} \\
H_{y r} & H_{y d_{\mathrm{i}}}
\end{array}\right] \\
& =\left[\begin{array}{cc}
C(I+P C)^{-1} & -C P(I+C P)^{-1} \\
P C(I+P C)^{-1} & P(I+C P)^{-1}
\end{array}\right] .
\end{aligned}
$$

Definition 2.1 The system $S(P, C)$ is:

- internally stable if every entry in the input-output transfer matrix $H$ is proper and stable, i.e. has all poles in $\mathbf{C}_{-}$, the open left half-plane;

- statically decoupled if it is internally stable and $H_{y r}$ is nonsingular and satisfies $H_{y r}(0)=I$;

- partially decoupled (or triangularly decoupled) if it is internally stable and $H_{y r}$ is nonsingular and lower left triangular; and

- diagonally decoupled if it is internally stable and $H_{y r}$ is nonsingular and diagonal.

These four properties form a hierarchy of control objectives. Internal stability is the most basic requirement of the closed-loop system $S(P, C)$; it ensures that all bounded signals $r, d_{\mathrm{i}}$ generate bounded responses at any other point in the loop. If $S(P, C)$ is statically decoupled then every component of the plant output $y$ is able to track step changes in the corresponding component of the reference input $r$ with asymptotically zero error. If $S(P, C)$ is partially decoupled, then the first $i-1$ components of the plant output vector $y$ are unaffected by variations in the $i$ th and subsequent components of $r$. Thus by designing to achieve partial decoupling of $S(P, C)$, a mechanism is provided for ranking plant output components according to their relative importance. Diagonally decoupling $S(P, C)$ ensures that each component of $y$ is affected only by the corresponding component of $r$.

In this paper we are concerned with obtaining controllers $C(s)$ for which $S(P, C)$ is internally stable, statically decoupled and at least partially decoupled. The design method to be proposed achieves diagonal decoupling as a special case of partial decoupling. 
By defining

$$
Q \triangleq C(I+P C)^{-1},
$$

the closed-loop transfer matrix $H_{y r}$ of $S(P, C)$ is given by

$$
H_{y r}=P Q
$$

The importance of $Q$ follows from the fact that, since $P$ is stable, $S(P, C)$ is internally stable if and only if $Q$ is stable, see e.g. (Morari and Zafiriou 1989 , p. 295). Moreover, since $P$ is strictly proper, $C$ defined by

$$
C \triangleq Q(I-P C)^{-1}
$$

is proper if and only if $Q$ is proper (Callier and Desoer 1982, p. 222). Thus the design problem we consider in this paper consists of obtaining a proper and stable $Q$ such that $H_{y r}=P Q$ is nonsingular and at least lower triangular.

Having obtained a suitable $Q$, the controller can be implemented using (2) to compute $C$. An alternative is to use $Q$ directly in the controller implementation using the Internal Model Control (IMC) feedback configuration (Morari and Zafiriou 1989).

The closed-loop transfer function $H_{y r}=P Q$ suggests that "perfect control" (i.e. $e(t)=0$ for all time $t$ and all inputs $r$ and $d_{\mathrm{i}}$ ) could be achieved by the choice

$$
Q=P^{-1} \text {. }
$$

It is useful to distinguish between two fundamental reasons why perfect control is never practical:

i) plant-limited design: If $P$ contains non-minimum phase zeros, then these appear as unstable poles of $Q$ since $\mathcal{Z}[P]=\mathcal{Z}\left[Q^{-1}\right]=$ $\mathcal{P}[Q]$. If $P$ contains pure time delays then $Q=P^{-1}$ is noncausal and cannot be used as a controller. Finally, since $P$ is strictly proper, $Q=P^{-1}$ is improper.

ii) knowledge-limited design: The plant model $P$ is only ever an approximate description of a physical process. In general the fidelity of the model decreases with increasing frequency and a limited closed-loop bandwidth is necessary for robust stability (Morari and Zafiriou 1989).

In this paper we focus on plant-limited design, though the tuning of the proposed controller will in practice be dictated by some specification of model uncertainty. 


\section{Design for decoupling: minimum phase plant}

This section deals with the limitations to perfect control imposed by the "zeros at infinity" of the plant $P$ which arise due to the strictly proper form of $P$. The results obtained then form a useful prototype for the consideration in the following section of the control limitations imposed by zeros in the closed-left half-plane $\mathbf{C}_{+} \triangleq\{s \in \mathbf{C} \mid \operatorname{Re}(s) \geq 0\}$.

For single-input single-output (SISO) systems, the notion of relative degree of a plant $p(s) \in \mathbf{R}_{\mathrm{sp}}(s)$ is useful in characterizing the class of closedloop transfer functions achievable via a proper compensator $c(s)$. For $p(s)=$ $n(s) / d(s)$, where $n(s), d(s) \in \mathbf{R}[s]$, the relative degree of $p(s)$ is defined as $\operatorname{deg} d(s)-\operatorname{deg} n(s)$. It then follows from the SISO version of (1) that all closed-loop transfer functions achievable by a proper compensator $q(s)$ have relative degree at least that of $p(s)$; a relative degree any lower would require an improper $q(s)$.

Knowledge of the relative degree of a scalar transfer function $p(s) \in$ $\mathbf{R}_{\mathrm{sp}}(s)$ is equivalent to knowledge of a polynomial $\xi(s)$ such that

$$
\lim _{s \rightarrow \infty} \xi(s) p(s)=k \neq 0
$$

where $\operatorname{deg} \xi(s)=\operatorname{deg} d(s)-\operatorname{deg} n(s)$, and $k$ is called the high frequency gain of the plant $p(s)$.

A natural extension of this idea to a MIMO plant $P(s) \in \mathbf{R}_{\mathrm{sp}}^{m \times m}$ is provided by a polynomial matrix $\xi(s)$ such that

$$
\lim _{s \rightarrow \infty} \xi(s) P(s)=K
$$

where $K \in \mathbf{R}^{m \times m}$ is nonsingular and called the high-frequency gain matrix. One particular form for $\xi(s)$ was presented by Wolovich and Falb (1976) and termed the "interactor matrix". The following Lemma details a slight modification of the interactor matrix such that $\xi^{-1}(s)$ is asymptotically stable and $\xi^{-1}(0)=I$. Similar modifications of the interactor have been proposed by Elliott and Wolovich (1982). The reasons for this normalization will be given shortly.

\section{Lemma 3.1 : Normalized interactor matrix}

Let $P(s) \in \mathbf{R}_{\mathrm{sp}}^{m \times m}(s)$ be stable and nonsingular, and let $a_{\mathrm{n}}>0$ be fixed. Then there is a unique, nonsingular, lower left triangular polynomial matrix $\xi_{\mathrm{n}}(s)$, known as the normalized interactor matrix, of the form

$$
\xi_{\mathrm{n}}(s)=\Sigma_{\mathrm{n}}(s) \Delta_{\mathrm{n}}(s),
$$


where

$$
\begin{aligned}
\Delta_{\mathrm{n}}(s)= & \operatorname{diag}\left[\left(a_{\mathrm{n}} s+1\right)^{n_{1}}, \cdots,\left(a_{\mathrm{n}} s+1\right)^{n_{m}}\right], \\
\Sigma_{\mathrm{n}}(s)= & {\left[\begin{array}{ccccc}
1 & 0 & \cdots & \cdots & 0 \\
\sigma_{21}(s) & 1 & & & 0 \\
\sigma_{31}(s) & \sigma_{32}(s) & \ddots & & \vdots \\
\vdots & \vdots & & \ddots & \vdots \\
\sigma_{m 1}(s) & \sigma_{m 2}(s) & \cdots & \cdots & 1
\end{array}\right], }
\end{aligned}
$$

and $\sigma_{i j}(s) \in \mathbf{R}[s]$ is divisible by $s$ (or is zero) such that

$$
\xi_{\mathrm{n}}(s) P(s)=K_{\mathrm{n}}(s),
$$

where $K_{\mathrm{n}}(s) \in \mathbf{R}_{p}^{m \times m}(s)$ is biproper, and $\xi_{\mathrm{n}}(0)=I$.

Proof. Using a straightforward modification of (Wolovich and Falb 1976, Lemma 3.1) (see e.g. (Middleton and Goodwin 1990, p. 251)), it can be shown that there is a unique, nonsingular lower left triangular matrix $\tilde{\xi}(s)$ of the form

$$
\tilde{\xi}(s)=\tilde{\Sigma}(s) \tilde{\Delta}(s),
$$

where

$$
\tilde{\Delta}(s)=\operatorname{diag}\left[\left(a_{\mathrm{n}} s+1\right)^{n_{1}}, \cdots,\left(a_{\mathrm{n}} s+1\right)^{n_{m}}\right]
$$

and

$$
\tilde{\Sigma}(s)=\left[\begin{array}{ccccc}
1 & 0 & \cdots & \cdots & 0 \\
\tilde{\sigma}_{21}(s) & 1 & & & 0 \\
\tilde{\sigma}_{31}(s) & \tilde{\sigma}_{32}(s) & \ddots & & \vdots \\
\vdots & \vdots & & \ddots & \vdots \\
\tilde{\sigma}_{m 1}(s) & \tilde{\sigma}_{m 2}(s) & \cdots & \cdots & 1
\end{array}\right],
$$

with $\tilde{\sigma}_{i j}(s) \in \mathbf{R}[s]$ divisible by $a_{\mathrm{n}} s+1$ (or zero) such that

$$
\tilde{\xi}(s) P(s)=\tilde{K}(s)
$$

with $\tilde{K}(s)$ biproper. Now define

$$
\xi_{\mathrm{n}}(s) \triangleq \Sigma_{\mathrm{n}}(s) \Delta_{\mathrm{n}}(s),
$$

where

$$
\begin{aligned}
\Sigma_{\mathrm{n}}(s) & \triangleq(\tilde{\xi}(0))^{-1} \tilde{\Sigma}(s), \\
\Delta_{\mathrm{n}}(s) & \triangleq \tilde{\Delta}(s) .
\end{aligned}
$$


Clearly $\Delta_{\mathrm{n}}(s)$ has the required form. Since $(\tilde{\xi}(0))^{-1}$ is lower unit triangular, $\Sigma_{\mathrm{n}}(s)$ defined in (10) is a lower unit triangular polynomial matrix. Furthermore, since

$$
\Sigma_{\mathrm{n}}(0)=(\tilde{\Sigma}(0) \tilde{\Delta}(0))^{-1} \tilde{\Sigma}(0)=\tilde{\Delta}(0)=I,
$$

the strictly lower triangular entries of $\Sigma_{\mathrm{n}}(s)$ are divisible by $s$ (or are zero). Clearly, $K(s)=\xi_{\mathrm{n}}(s) P(s)$ is biproper since

$$
\begin{aligned}
\xi_{\mathrm{n}}(s) P(s) & =(\tilde{\xi}(0))^{-1} \tilde{\xi}(s) P(s) \\
& =(\tilde{\xi}(0))^{-1} \tilde{K}(s),
\end{aligned}
$$

and both $(\tilde{\xi}(0))^{-1}$ and $\tilde{K}(s)$ are biproper. Finally,

$$
\begin{aligned}
\xi_{\mathrm{n}}(0) & =\Sigma_{\mathrm{n}}(0) \Delta_{\mathrm{n}}(0) \\
& =(\tilde{\xi}(0))^{-1} \tilde{\Sigma}(0) \tilde{\Delta}(0) \\
& =(\tilde{\xi}(0))^{-1} \tilde{\xi}(0) \\
& =I .
\end{aligned}
$$

A procedure for constructing the interactor matrix is given in (Wolovich and Falb 1976). Furuta and Kamiyama (1977) give a method for computing the Wolovich-Falb interactor from a state-space representation of $P(s)$. Chang and Wang (1990) have presented an algorithm for computing the interactor matrix from a right matrix fraction description (RMFD) of the plant.

\subsection{Design for partial decoupling: minimum phase plant}

The interactor matrix provides a factorization of the plant model $P(s)$ into the product of two transfer matrices:

$$
P(s)=\xi_{\mathrm{n}}^{-1}(s) K_{\mathrm{n}}(s),
$$

where $\xi_{\mathrm{n}}^{-1}(s) \in \mathbf{R}_{\mathrm{sp}}^{m \times m}(s)$ contains the "strictly proper part" of $P(s)$ and $K_{\mathrm{n}}(s) \in \mathbf{R}_{\mathrm{p}}^{m \times m}(s)$ contains the invertible part. It is possible to utilize this factorization of $P(s)$ to design for partial decoupling by selecting

$$
Q(s)=K_{\mathrm{n}}^{-1}(s) .
$$

When $P$ is stable and minimum phase, $K_{\mathrm{n}}^{-1}(s)$ is proper and stable, and do design (13) ensures internal stability of $S(P, C)$. The resulting closed-loop 
transfer function given by

$$
\begin{aligned}
H_{y r}(s) & =P(s) Q(s) \\
& =\left(\xi_{\mathrm{n}}^{-1}(s) K_{\mathrm{n}}(s)\right) K_{\mathrm{n}}^{-1}(s) \\
& =\xi_{\mathrm{n}}^{-1}(s)
\end{aligned}
$$

is lower triangular, and since $\xi_{\mathrm{n}}(0)=I, S(P, C)$ is also statically decoupled.

Designing for partial decoupling in this fashion has an intuitive interpretation: $\xi_{\mathrm{n}}^{-1}(s)$ represents that part of the plant model which cannot be inverted since doing so would lead to an improper $Q(s)$. Selecting $Q(s)$ as the inverse of the invertible part of the plant as in (13) leads to the closed-loop transfer function (14) which clearly consists of the non-invertible component of $P(s)$.

Equation (14) also indicates why it is preferable for design purposes to use a normalized interactor as in Lemma 3.1 instead of the Wolovich-Falb interactor. Since $\xi_{\mathrm{n}}^{-1}(s)=\operatorname{adj}\left(\xi_{\mathrm{n}}(s)\right) / \operatorname{det}\left(\xi_{\mathrm{n}}(s)\right)$ and $\operatorname{det}\left(\xi_{\mathrm{n}}(s)\right)=\left(a_{\mathrm{n}} s+\right.$ $1)^{n_{1}+\cdots+n_{m}}, H_{y r}$ is stable. Furthermore, since $\xi_{\mathrm{n}}^{-1}(0)=I$, static decoupling of $S(P, C)$ is also achieved.

\subsection{Design for diagonal decoupling: minimum phase plant}

The normalized interactor matrix can also be used in designing for diagonal decoupling of $S(P, C)$ as follows. Consider the closed-loop transfer function arising from the (partially decoupled) design (13):

$$
\begin{aligned}
H_{y r} & =\xi_{\mathrm{n}}^{-1} \\
& =\Delta_{\mathrm{n}}^{-1} \Sigma_{\mathrm{n}}^{-1}
\end{aligned}
$$

Since $\Delta_{\mathrm{n}}^{-1}$ is diagonal, it is only the $\Sigma_{\mathrm{n}}^{-1}$ term which potentially prevents $H_{y r}$ from being diagonal. Choosing

$$
Q=K_{\mathrm{n}}^{-1} \Sigma_{\mathrm{n}}
$$

leads to

$$
\begin{aligned}
H_{y r} & =P Q \\
& =\left(\Sigma_{\mathrm{n}} \Delta_{\mathrm{n}}\right)^{-1} K_{\mathrm{n}} K_{\mathrm{n}}^{-1} \Sigma_{\mathrm{n}} \\
& =\Delta_{\mathrm{n}}^{-1},
\end{aligned}
$$

which is clearly diagonal. However $Q=K_{\mathrm{n}}^{-1} \Sigma_{\mathrm{n}}$ is improper since $K_{\mathrm{n}}$ is biproper and $\Sigma_{\mathrm{n}}$ is a polynomial matrix. What is required is a diagonal 
matrix $D_{\mathrm{n}} \in \mathbf{R}_{\mathrm{sp}}^{m \times m}(s)$ such that

$$
Q=K_{\mathrm{n}}^{-1} \Sigma_{\mathrm{n}} D_{\mathrm{n}}
$$

is proper, since then the closed-loop transfer function

$$
H_{y r}=\Delta_{\mathrm{n}}^{-1} D_{\mathrm{n}}
$$

would be diagonal. The following Lemma defines such a suitable matrix.

Lemma 3.2 Let $\xi_{\mathrm{n}}(s)=\Sigma_{\mathrm{n}}(s) \Delta_{\mathrm{n}}(s)$ be the normalized interactor matrix associated with $P(s) \in \mathbf{R}_{\mathrm{sp}}^{m \times m}(s)$, assumed stable and nonsingular, and let $a_{\mathrm{n}}$ be the design variable in $\xi_{\mathrm{n}}(s)$. Let $D_{\mathrm{n}}(s)$ be defined by

$$
\begin{aligned}
D_{\mathrm{n}}(s) & =\operatorname{diag}\left[D_{1}(s), \cdots, D_{m}(s)\right] \\
D_{j}(s) & =\left(a_{\mathrm{n}} s+1\right)^{-n_{j}}, \quad j=1, \ldots, m \\
n_{j} & \triangleq \partial c_{j}\left(\Sigma_{\mathrm{n}}(s)\right), \quad j=1, \ldots, m .
\end{aligned}
$$

That is, $n_{j}$ is the maximum polynomial degree of $s$ in the $j$ th column of $\Sigma_{n}(s)$, and $n_{j} \triangleq 0$ if the jth column of $\Sigma_{\mathrm{n}}(s)$ is independent of $s$. Then

i) $D_{\mathrm{n}}(s)$ is such that $\Sigma_{\mathrm{n}}(s) D_{\mathrm{n}}(s)$ is proper and stable; and

ii) $D_{\mathrm{n}}(0)=I$.

Proof. Since $\Sigma_{\mathrm{n}}(s)$ is unit lower triangular with all strictly lower triangular entries being polynomials in $s$ either divisible by $s$ or identically zero, the first column of $\Sigma_{\mathrm{n}}(s) D_{\mathrm{n}}(s)$ has the form

$$
\left[D_{1}(s), \sigma_{2,1}(s) D_{1}(s), \sigma_{3,1}(s) D_{1}(s), \cdots, \sigma_{m, 1}(s) D_{1}(s)\right]^{T},
$$

where $\sigma_{j, 1}(s) \in \mathbf{R}[s]$ are either identically zero or of degree at most $n_{1}$. Since $D_{1}(s)=\left(a_{\mathrm{n}} s+1\right)^{-n_{1}}$, it follows that each of the terms in the first column of $\Sigma_{\mathrm{n}}(s) D_{\mathrm{n}}(s)$ is proper and stable. Moreover, since each strictly lower triangular entry of $\Sigma_{n}(s)$ is divisible by $s$ (or is zero), the corresponding terms in $\Sigma_{\mathrm{n}}(s) D_{1}(s)$ evaluate to zero at $s=0$, and since $D_{1}(0)=1$, it follows that the first column of $\Sigma_{\mathrm{n}}(0) D_{\mathrm{n}}(0)$ has the form $[1,0, \cdots, 0]^{T}$. Analogous arguments for columns $2,3, \cdots, m$ of $\Sigma_{\mathrm{n}}(s) D_{\mathrm{n}}(s)$ give the result.

With these preliminaries, the key properties of a design for diagonal decoupling of a minimum phase plant are summarized in the following Lemma. 


\section{Lemma 3.3 : Design for diagonal decoupling (minimum phase plant)}

Let $P(s) \in \mathbf{R}_{\mathrm{sp}}^{m \times m}(s)$ be nonsingular, stable and minimum phase, let $\xi_{\mathrm{n}}(s)$ be the normalized interactor associated with $P(s)$ :

$$
P=\xi_{\mathrm{n}}^{-1} K_{\mathrm{n}}
$$

and let $D_{\mathrm{n}}$ be the diagonal transfer matrix defined in Lemma 3.2. Then the design

$$
Q=K_{\mathrm{n}}^{-1} \Sigma_{\mathrm{n}} D_{\mathrm{n}}
$$

achieves both static and diagonal decoupling (and hence internal stability) of $S(P, C)$ such that

$$
H_{y r}=\Delta_{\mathrm{n}}^{-1} D_{\mathrm{n}}
$$

Moreover, no proper compensator can achieve a lower relative degree of any entry in $H_{y r}$ than that achieved in (16).

Proof.

$$
\begin{aligned}
H_{y r} & =P Q \\
& =\xi^{-1} K_{\mathrm{n}} Q \\
& =\left(\Sigma_{\mathrm{n}} \Delta_{\mathrm{n}}\right)^{-1} K_{\mathrm{n}} K_{\mathrm{n}}^{-1} \Sigma_{\mathrm{n}} D_{\mathrm{n}} \\
& =\Delta_{\mathrm{n}}^{-1} D_{\mathrm{n}} .
\end{aligned}
$$

From Lemma 3.2, $\Sigma_{\mathrm{n}} D_{\mathrm{n}}$ is proper and stable, and since $K_{\mathrm{n}}^{-1}$ is proper and stable, it follows that $Q$ is proper and stable; hence $S(P, C)$ is internally stable. By the construction of $\Delta_{\mathrm{n}}$ and $D_{\mathrm{n}}$ in Lemmas 3.1 and 3.2 respectively, $H_{y r}(0)=\Delta_{\mathrm{n}}^{-1}(0) D_{\mathrm{n}}(0)=I$. Thus $S(P, C)$ is statically decoupled, and since both $\Delta_{\mathrm{n}}^{-1}(s)$ and $D_{\mathrm{n}}(s)$ are diagonal, $S(P, C)$ is diagonally decoupled also.

Suppose a compensator $\tilde{Q}$ achieves

$$
H_{y r}=\Delta_{\mathrm{n}}^{-1} \tilde{D}
$$

for some $\tilde{D}=\operatorname{diag}\left[\tilde{D}_{1}, \cdots, \tilde{D}_{m}\right]$ with entry $\tilde{D}_{i}$ of strictly lower relative degree than the corresponding entry $D_{i}$ of $D_{\mathrm{n}}=\operatorname{diag}\left[D_{1}, \cdots, D_{m}\right]$ defined in Lemma 3.2. The definition of $D$ is such that the entries in the column vector $\Sigma_{\mathrm{n}} D_{i}$ corresponding to the highest polynomial degree of $s$ in the $i$ th column of $\Sigma_{\mathrm{n}}$ will be proper, but not strictly proper, transfer functions. Thus if the relative degree of $\tilde{D}_{i}$ is strictly less then the relative degree of $D_{i}$, the column vector $\Sigma_{\mathrm{n}} \tilde{D}_{i}\left(\right.$ and hence $\left.\tilde{Q}=K_{\mathrm{n}}^{-1} \Sigma_{\mathrm{n}} \tilde{D}\right)$ will be improper.

Lemma 3.3 makes explicit the cost of decoupling stable minimum phase plants. Unless the normalized interactor matrix is diagonal (and thus $\Sigma_{\mathrm{n}}=$ 
$I)$, the additional terms in $D_{\mathrm{n}}$ required to ensure $\Sigma_{\mathrm{n}} D_{\mathrm{n}}$ is proper appear directly in the closed-loop transfer function (16). These strictly proper terms in $D_{\mathrm{n}}$ are regarded as a cost in terms of the speed of closed-loop response. That is, the rise time of a step response for a system with transfer function $(a s+1)^{-p}$ exceeds that for the system $(a s+1)^{-q}$ for $p>q$.

The following Corollary indicates that the smallest achievable relative degrees in the entries of $H_{y r}$ can be read directly from the normalized interactor matrix.

Corollary 3.1 Let $H_{y r}=\operatorname{diag}\left[\left(a_{\mathrm{n}} s+1\right)^{-p_{1}}, \cdots,\left(a_{\mathrm{n}} s+1\right)^{-p_{m}}\right]$ be the diagonal closed-loop transfer function of $S(P, C)$ achieved by the design (15) of Lemma 3.3. Then the $p_{i}$ are given by

$$
p_{i}=\partial c_{i}\left(\xi_{\mathrm{n}}\right)=i \text { th column degree of } \xi_{\mathrm{n}}, \quad i=1,2, \cdots, m \text {. }
$$

Proof. From Lemma 3.3, the diagonal closed-loop transfer function is given by

$$
H_{y r}=\Delta_{\mathrm{n}}^{-1} D_{\mathrm{n}}
$$

Thus

$$
\begin{aligned}
p_{1} & =\partial\left(\Delta_{1}\right)+\partial\left(D_{1}^{-1}\right) \\
& =\partial\left(\Delta_{1}\right)+\partial c_{1}\left(\Sigma_{\mathrm{n}}\right) \quad \text { using the definition of } D_{1} \text { in Lemma } 3.2 \\
& =\partial c_{1}\left(\xi_{\mathrm{n}}\right)
\end{aligned}
$$

Analogous arguments for $p_{2}$ through $p_{m}$ give the result.

\section{Design for decoupling: non-minimum phase plants}

In the previous section the normalized interactor matrix was defined and shown to be useful in designing stabilizing compensators which achieve static and either partial or diagonal decoupling for minimum phase plants. The key to these designs is that the normalized interactor provides a factorization of $P$ :

$$
P=\xi_{\mathrm{n}}^{-1} K_{\mathrm{n}}
$$

in which $\xi_{\mathrm{n}}^{-1}$ contains the strictly proper (i.e. non-invertible) component of $P$ and $K_{\mathrm{n}}$ is biproper-bistable (i.e. both $K_{\mathrm{n}}$ and $K_{\mathrm{n}}^{-1}$ are proper and stable transfer matrices). This factorization leads to internally stabilizing controllers since the zeros at infinity extracted from $P$ by $\xi_{n}^{-1}$ are the only 
impediment to perfect control if complete knowledge of the plant model is assumed.

If $P$ contains non-minimum phase zeros (i.e. zeros in $\mathbf{C}_{+}$), then the designs of the previous section will fail to stabilize $S(P, C)$ since the right half-plane zeros of $P$ appear as unstable poles of $Q: \mathcal{P}[Q]=\mathcal{P}\left[K_{\mathrm{n}}^{-1}\right]=\mathcal{Z}\left[K_{\mathrm{n}}\right]=\mathcal{Z}[P]$.

In this section we present a generalization of the results of Section 3 to provide stable decoupling designs for non-minimum phase plants. The presentation here follows closely that of Tsiligiannis and Svoronos (1989), who developed a generalized interactor for discrete-time systems. The generalized interactor here is in fact a special case of the left structure matrix in $\overline{\mathbf{C}}_{+}$of the plant $P$, where $\overline{\mathbf{C}}_{+} \triangleq \mathbf{C}_{+} \cup\{\infty\}$ (Vardulakis 1991). Its normalized and lower triangular form simplify design for static and partial decoupling, and design freedom is captured in a small number of scalar parameters.

Since the normalized interactor matrix extracts zeros at $s=\infty$ from $P(s)$, it is possible to extract a finite right half-plane zero $z$ from $P(s)$ by introducing a transformation of the form

$$
s^{\prime}=\frac{s}{s-z}
$$

to transform a zero at $s=z$ to a zero at $s^{\prime}=\infty$, then computing the normalized interactor of the transformed system. Some scaling of the transformation (17) is necessary to ensure $\xi(0)=I$, the exact nature of which is made precise in the following Lemma.

\section{Lemma 4.1 : z-interactor matrix}

Let $P(s) \in \mathbf{R}_{\mathrm{sp}}^{m \times m}(s)$ be stable and nonsingular, let $a>0$ be fixed, and let $z$ be a zero of $P(s)$ from the open right-half plane. The there is a unique, lower left triangular rational matrix $\xi_{z}$, called the $z$-interactor matrix, of the form

$$
\xi_{z}(s)=\Sigma_{z}\left(s^{\prime}\right) \Delta_{z}\left(s^{\prime}\right),
$$

where

$$
\begin{gathered}
\Delta_{z}\left(s^{\prime}\right)=\operatorname{diag}\left[\left(a s^{\prime}+1\right)^{n_{1}}, \cdots,\left(a s^{\prime}+1\right)^{n_{m}}\right], \\
\Sigma_{z}\left(s^{\prime}\right)=\left[\begin{array}{ccccc}
1 & 0 & \cdots & \cdots & 0 \\
\sigma_{21}^{z}\left(s^{\prime}\right) & 1 & & & 0 \\
\sigma_{31}^{z}\left(s^{\prime}\right) & \sigma_{32}^{z}\left(s^{\prime}\right) & \ddots & & \vdots \\
\vdots & \vdots & & \ddots & \vdots \\
\sigma_{m 1}^{z}\left(s^{\prime}\right) & \sigma_{m 2}^{z}\left(s^{\prime}\right) & \cdots & \cdots & 1
\end{array}\right], \\
s^{\prime}=\frac{-s}{s-z}\left(\frac{1+a z}{a}\right),
\end{gathered}
$$

and $\sigma_{i j}^{z}\left(s^{\prime}\right)$ are polynomials in $s^{\prime}$ divisible by $s^{\prime}$ (or zero) such that: 
i)

$$
\lim _{s \rightarrow z} \xi_{z}(s) P(s)=K_{z}
$$

where $K_{z} \in \mathbf{R}^{m \times m}$ is nonsingular;

ii) $\xi_{z}^{-1}(s)$ is proper and stable;

iii) $\xi_{z}(s)$ removes the right half-plane zero z from $P(s)$ :

$$
\mathcal{Z}\left[\xi_{z}(s) P(s)\right] \cap \mathbf{C}_{+}=\left(\mathcal{Z}[P(s)] \cap \mathbf{C}_{+}\right) \backslash\{z\} ;
$$

iv) $\xi_{z}(0)=I$.

Proof. i) From (21),

so if

$$
s=\frac{a s^{\prime} z}{s^{\prime} a+1+a z}
$$

$$
P\left(s^{\prime}\right)=\left.P(s)\right|_{s=\frac{a s^{\prime} z}{s^{\prime} a+1+a z}}
$$

then $\xi_{z}$, the $z$-interactor of $P(s)$, is equal to $\xi$, the normalized interactor of $P\left(s^{\prime}\right)$. Thus

$$
\begin{aligned}
\lim _{s \rightarrow z} \xi_{z}(s) P(s) & =\lim _{s^{\prime} \rightarrow \infty} \xi\left(s^{\prime}\right) P\left(s^{\prime}\right) \\
& =\lim _{s^{\prime} \rightarrow \infty} K\left(s^{\prime}\right)
\end{aligned}
$$

which is nonsingular since $K(s)$ is biproper.

ii) Since $\xi_{z}$ is polynomial in $s^{\prime}, \xi_{z}^{-1}$ is polynomial in $\left(s^{\prime}\right)^{-1}$, and since $\operatorname{det} \xi_{z}=\left(a s^{\prime}+1\right)^{n_{1}+\cdots n_{m}}$ with

$$
\left(a s^{\prime}+1\right)^{-1}=\frac{z-s}{z(a s+1)},
$$

it follows that $\xi_{z}^{-1}(s)$ is stable. Moreover, $\xi_{z}^{-1}(s)$ is proper since $\xi_{z}^{-1}(s)$ is polynomial in $(z-s) \backslash(z(a s+1))$ and

$$
\lim _{s \rightarrow \infty} \frac{z-s}{z(a s+1)}=\frac{-1}{a z}
$$

iii) From part ii), $\xi_{z}^{-1}(s)$ is stable, and so

$$
\begin{aligned}
\mathcal{Z}\left[\xi_{z}\right] & =\mathcal{P}\left[\xi_{z}^{-1}\right] \\
& =\emptyset .
\end{aligned}
$$

Hence $\xi_{z}$ contributes no zeros in $\mathbf{C}_{+}$. The result follows upon noting that $z \notin \mathcal{Z}\left[\xi_{z} P\right]$ since from part i), $\lim _{s \rightarrow z} \xi_{z} P$ is nonsingular.

iv) Immediate from (19), (20), (21).

To compute the $z$-interactor of $P(s)$ for some particular $z$, the procedure is as follows: 


\section{Step 1.}

Compute $P\left(s^{\prime}\right)$ by substituting

$$
s=\frac{a s^{\prime} z}{s^{\prime} a+1+a z}
$$

into the expression for $P(s)$.

\section{Step 2.}

Compute the normalized interactor $\xi\left(s^{\prime}\right)$ of $P\left(s^{\prime}\right)$.

\section{Step 3.}

Substitute the expression (21) for $s^{\prime}$ into $\xi\left(s^{\prime}\right)$ to obtain $\xi_{z}(s)$.

It is apparent from part iii) of Lemma 4.1 that the $z$-interactor extracts only one right half-plane zero from $P(s)$. To extract all the right half-plane zeros from $P(s)$, together with the zeros at $s=\infty$ extracted by the normalized interactor, we define the generalized interactor matrix as follows.

\section{Definition 4.1 : Generalized interactor matrix}

Let $\left\{z_{1}, z_{2}, \cdots, z_{p}\right\}$ be the set of all zeros of $P(s)$ in the right half-plane. Then the generalized interactor matrix $\xi(s)$ of $P(s)$ is defined:

$$
\xi(s) \triangleq \xi_{z_{p}}(s) \xi_{z_{p-1}}(s) \cdots \xi_{z_{1}}(s) \xi_{n}(s)
$$

where $\xi_{\mathrm{n}}(s)$ is the normalized interactor of $P(s), \xi_{z_{i}}(s)$ is the $z_{i}$-interactor of $K_{i}(s)$ :

$$
K_{i+1}(s)=\xi_{z_{i}}(s) K_{i}(s), \quad i=1,2, \ldots p
$$

and

$$
K_{1}(s)=\xi_{\mathrm{n}}(s) P(s)
$$

The following Lemma asserts that the generalized interactor extracts the entire non-invertible part of $P$.

\section{Lemma 4.2 : Properties of the generalized interactor matrix}

Let $P(s) \in \mathbf{R}_{\mathrm{sp}}^{m \times m}(s)$ be stable and nonsingular, and let $\left\{z_{1}, z_{2}, \cdots, z_{p}\right\}$ be the set of all zeros of $P(s)$ in the right half-plane. Then the generalized interactor matrix $\xi(s)$ has the properties:

i) $\lim _{s \rightarrow z} \xi(s) P(s)$ is finite and nonsingular for all $z \in\left\{z_{1}, z_{2}, \cdots, z_{p}, \infty\right\}$; 
ii)

$$
\xi(s) P(s)=K(s)
$$

where $K^{-1}(s)$ is proper and stable; and

iii) $\xi(0)=I$.

Proof. i) If $z=\infty$ then

$$
\lim _{s \rightarrow z} \xi(s) P(s)=\lim _{s \rightarrow \infty}\left[\xi_{z_{p}}(s) \cdots \xi_{z_{1}}(s)\right] \lim _{s \rightarrow \infty}\left[\xi_{\mathrm{n}}(s) P(s)\right],
$$

which is finite and nonsingular since both terms on the right-hand-side are. Likewise if $z=z_{i} \in\left\{z_{1}, \cdots, z_{p}\right\}$ then

$$
\lim _{s \rightarrow z} \xi(s) P(s)=\lim _{s \rightarrow z_{i}}\left[\xi_{z_{p}}(s) \cdots \xi_{z_{i+1}}(s)\right] \lim _{s \rightarrow z_{i}}\left[\xi_{z_{i}}(s) K_{i}(s)\right]
$$

is finite and nonsingular.

ii) From i), $\xi(s) P(s)$ has no zeros in $\left\{z_{1}, z_{2}, \cdots, z_{p}, \infty\right\}$. Thus $K^{-1}=$ $(\xi P)^{-1}$ has no poles in $\left\{z_{1}, z_{2}, \cdots, z_{p}, \infty\right\}$. Therefore $K^{-1}$ is proper, since $K^{-1}$ has no poles at $s=\infty$, and stable since $\xi^{-1}$ has no unstable poles except for those in $\left\{z_{1}, z_{2}, \cdots, z_{p}\right\}$.

iii) Immediate since $\xi_{z_{p}}(0)=\cdots=\xi_{z_{1}}(0)=\xi_{\mathrm{n}}(0)=I$.

\subsection{Design for partial decoupling: non-minimum phase plant}

The factorization of the plant model $P(s)$ provided by the generalized interactor of Lemma 4.2 can be used to design for partial decoupling as shown in the following Lemma.

\section{Lemma 4.3 : Design for partial decoupling (non-minimum phase} plant)

Let $P(s) \in \mathbf{R}_{\mathrm{sp}}^{m \times m}(s)$ be nonsingular and stable, and let $\xi(s)$ be the generalized interactor associated with $P(s)$ :

$$
P=\xi^{-1} K
$$

Then the design

$$
Q=K^{-1}
$$

achieves both static and partial decoupling (and hence internal stability) of $S(P, C)$ such that

$$
H_{y r}=\xi^{-1} \text {. }
$$


Proof. From Lemma 4.2, $Q=K^{-1}$ is proper and stable, hence $S(P, C)$ is internally stable. The closed-loop transfer function is given by

$$
\begin{aligned}
H_{y r} & =P Q \\
& =\left(\xi^{-1} K\right) K^{-1} \\
& =\xi^{-1}
\end{aligned}
$$

and since $\xi^{-1}(s)$ is lower triangular and satisfies $\xi^{-1}(0)=I, S(P, C)$ is both statically and partially decoupled.

\subsection{Design for diagonal decoupling: non-minimum phase plant}

The same line of reasoning as in Section 3 will be pursued here to achieve diagonal decoupling of $S(P, C)$ when $P$ is no longer minimum phase. In this case, the generalized interactor $\xi$ provides a factorization of $\mathrm{P}$ :

$$
P=\xi^{-1} K
$$

with $K^{-1}$ biproper-bistable. From Definition 4.1, $\xi$ is constructed as follows:

$$
\begin{aligned}
\xi & =\xi_{z_{p}} \cdots \xi_{z_{1}} \xi_{\mathrm{n}} \\
& =\left(\Sigma_{z_{p}} \Delta_{z_{p}}\right) \cdots\left(\Sigma_{z_{1}} \Delta_{z_{1}}\right)\left(\Sigma_{\mathrm{n}} \Delta_{\mathrm{n}}\right) .
\end{aligned}
$$

To maintain the analogy with the design for diagonal decoupling when $P$ is minimum phase, it is desirable that $\xi$ be written as a product

$$
\xi=\Sigma \Delta
$$

where $\Sigma$ is unit lower triangular and $\Delta$ is diagonal. The following Lemma is useful in achieving such a factorization of $\xi$, which is complicated by the fact that the matrix multiplications in (22) are non-commutative.

Lemma 4.4 Let $\Sigma(s)$ be a unit lower triangular matrix:

$$
\Sigma(s)=\left[\begin{array}{ccccc}
1 & 0 & \cdots & \cdots & 0 \\
\sigma_{21}(s) & 1 & & & 0 \\
\sigma_{31}(s) & \sigma_{32}(s) & \ddots & & \vdots \\
\vdots & \vdots & & \ddots & \vdots \\
\sigma_{m 1}(s) & \sigma_{m 2}(s) & \cdots & \cdots & 1
\end{array}\right]
$$

and let

$$
\Delta(s)=\operatorname{diag}\left[\delta_{1}(s), \cdots, \delta_{m}(s)\right]
$$


where $\sigma_{i j}(s), \delta_{i}(s)$ are rational functions of $s$, not necessarily proper, and $\delta_{i}(s) \not \equiv 0$. Then

$$
\Delta \Sigma=\tilde{\Sigma} \Delta
$$

where

$$
\tilde{\Sigma}=\left[\begin{array}{ccccc}
1 & 0 & \cdots & \cdots & 0 \\
\frac{\delta_{2} \sigma_{21}}{\delta_{1}} & 1 & & & 0 \\
\frac{\delta_{3} \sigma_{31}}{\delta_{1}} & \frac{\delta_{3} \sigma_{32}}{\delta_{2}} & \ddots & & \vdots \\
\vdots & \vdots & & \ddots & \vdots \\
\frac{\delta_{m} \sigma_{m 1}}{\delta_{1}} & \frac{\delta_{m} \sigma_{m 2}}{\delta_{2}} & \cdots & \cdots & 1
\end{array}\right]
$$

Proof. Immediate since $\delta_{i}(s) \not \equiv 0$ ensures that the divisions by $\delta_{i}(s)$ are well-defined.

As shown in the following Lemma, repeated applications of Lemma 4.4 enable the generalized interactor matrix $\xi$ to be manipulated into the form (23) suitable for design purposes.

Lemma 4.5 : Factorizing the generalized interactor: $\xi=\Sigma \Delta$

Let $P(s) \in \mathbf{R}_{\mathrm{sp}}^{m \times m}(s)$ be nonsingular and stable. Let $\xi(s)$ be the generalized interactor matrix associated with $P(s)$ :

$$
P=\xi^{-1} K
$$

where

$$
\begin{aligned}
\xi & =\xi_{z_{p}} \cdots \xi_{z_{1}} \xi_{\mathrm{n}} \\
& =\left(\Sigma_{z_{p}} \Delta_{z_{p}}\right) \cdots\left(\Sigma_{z_{1}} \Delta_{z_{1}}\right)\left(\Sigma_{\mathrm{n}} \Delta_{\mathrm{n}}\right) .
\end{aligned}
$$

Then $\xi$ can be expressed in the form

$$
\xi=\Sigma \Delta
$$

where $\Sigma$ is unit lower triangular and

$$
\Delta \triangleq \Delta_{z_{p}} \cdots \Delta_{z_{1}} \Delta_{\mathrm{n}}
$$

Proof. Using Lemma 4.4,

$$
\begin{aligned}
\left(\Sigma_{z_{p}} \Delta_{z_{p}}\right)\left(\Sigma_{z_{p-1}} \Delta_{z_{p-1}}\right) & =\Sigma_{z_{p}} \tilde{\Sigma}_{z_{p-1}} \Delta_{z_{p}} \Delta_{z_{p-1}} \\
& =\Sigma^{1} \Delta^{1}
\end{aligned}
$$




\subsection{Design for diagonal decoupling: non-minimum phase plant20}

where $\Sigma^{1} \triangleq \Sigma_{z_{p}} \tilde{\Sigma}_{z_{p-1}}$ is unit lower triangular since both $\Sigma_{z_{p}}, \tilde{\Sigma}_{z_{p-1}}$ are, and $\Delta^{1} \triangleq \Delta_{z_{p}} \Delta_{z_{p-1}}$. Thus

$$
\begin{aligned}
\xi & =\left(\Sigma^{1} \Delta^{1}\right)\left(\Sigma_{z_{p-2}} \Delta_{z_{p-2}}\right) \cdots\left(\Sigma_{z_{1}} \Delta_{z_{1}}\right)\left(\Sigma_{\mathrm{n}} \Delta_{\mathrm{n}}\right) \\
& =\left(\Sigma^{2} \Delta^{2}\right)\left(\Sigma_{z_{p-3}} \Delta_{z_{p-3}}\right) \cdots\left(\Sigma_{z_{1}} \Delta_{z_{1}}\right)\left(\Sigma_{\mathrm{n}} \Delta_{\mathrm{n}}\right)
\end{aligned}
$$

upon applying Lemma 4.4 to $\Sigma^{1} \Delta^{1} \Sigma_{z_{p-2}} \Delta_{z_{p-2}}$. Continuing in this fashion yields the result.

Suppose now that the generalized interactor of some non-minimum phase plant $P$ has been computed, and factorized as in Lemma 4.5. Then the design

$$
Q=K^{-1} \Sigma
$$

achieves a closed-loop transfer function $H_{y r}=\Delta^{-1}$ which is diagonal. However $Q$ chosen in this fashion fails to internally stabilize $S(P, C)$ since entries of $\Sigma$ (and hence $Q$ ) will be unstable and/or improper. As in Section 3, we require a diagonal matrix $D$ such that $\Sigma D$, and hence $Q=K^{-1} \Sigma D$, is proper and stable. The following Definition supplies such a matrix.

Definition 4.2 Let $\xi(s)=\Sigma(s) \Delta(s)$ be the generalized interactor matrix associated with $P(s) \in \mathbf{R}_{\mathrm{sp}}^{m \times m}(s)$, assumed stable and nonsingular. Then let $D(s)$ be defined by

$$
D(s) \triangleq \operatorname{diag}\left[D_{1}(s), \cdots, D_{m}(s)\right]
$$

where

$D_{j}(s) \triangleq\left(\frac{z_{p}-s}{z_{p}\left(a_{p} s+1\right)}\right)^{n_{p, j}} \cdots\left(\frac{z_{1}-s}{z_{1}\left(a_{1} s+1\right)}\right)^{n_{1, j}}\left(\frac{1}{a_{\mathrm{n}} s+1}\right)^{n_{n, j}} \quad j=1, \cdots, m$ and $n_{p, j} \cdots n_{1, j}, n_{n, j}$ for $j=1, \cdots, m$ are the smallest integers necessary to ensure the $j$ th column of $\Sigma(s) D(s)$ is both proper and stable.

From this Definition, it is apparent that a reasonable choice for the design variables $a_{i}$ is:

$$
a_{i}=\frac{1}{z_{i}}, \quad i=1, \ldots, p,
$$

since this ensures that every right half-plane zero appearing on the diagonal of the resulting closed-loop transfer matrix $H_{y r}$ appears as part of an allpass factor. Using Definition 4.2, we can now state the following Lemma, in which the key properties of a design for diagonal decoupling of a nonminimum phase plant are summarized. 
Lemma 4.6 : Design for diagonal decoupling (non-minimum phase plant)

Let $P(s) \in \mathbf{R}_{\mathrm{sp}}^{m \times m}(s)$ be nonsingular and stable, let $\xi(s)$ be the generalized interactor associated with $P(s)$ :

$$
P=\xi^{-1} K
$$

and let $D$ be the diagonal transfer matrix defined in Definition 4.2. Then the design

$$
Q=K^{-1} \Sigma D
$$

achieves both static and diagonal decoupling (and hence internal stability) of $S(P, C)$ such that

$$
H_{y r}=\Delta^{-1} D
$$

Proof. For the proof that $H_{y r}=\Delta^{-1} D$, see the proof of Lemma 3.3. By definition of $D, \Sigma D$ is proper and stable, and since from part ii) of Lemma 4.2, $K^{-1}$ is proper and stable, it follows that $Q$ is proper and stable; hence $S(P, C)$ is internally stable. $\Delta(0)=\Delta_{z_{p}}(0)=\cdots=\Delta_{z_{1}}(0)=\Delta_{\mathrm{n}}(0)=I$ and from Definition 4.2, $D(0)=I$. Thus $H_{y r}(0)=I$ and $S(P, C)$ is statically decoupled. Since both $\Delta(s)$ and $D(s)$ are diagonal, $S(P, C)$ is diagonally decoupled also.

It is possible to use $\xi$ directly, as in Corollary 3.1, to infer the zeros from $\overline{\mathbf{C}}_{+}$which must appear in any diagonal closed-loop transfer function obtained by a controller $Q$ which internally stabilizes $S(P, C)$, see for example (Tsiligiannis and Svoronos 1989, Theorem 7). The approach of this section has been to utilize the factorization $\xi=\Sigma D$ to identify a diagonal transfer matrix $D$ such that $Q=K^{-1} \Sigma D$ is proper and stable, and which yields the closed-loop transfer function $H_{y r}=\Delta^{-1} D$. It is this latter approach which leads to a unified treatment of design for partial and diagonal decoupling in the following section.

\section{$5 \quad$ Blending designs for diagonal and partial decoupling}

In the previous section, controller design procedures were presented which achieve internal stability, static decoupling and either partial or diagonal decoupling of the closed-loop system $S(P, C)$. Associated with both the partially and diagonally decoupled designs are performance characteristics which may be undesirable. For example, the partially decoupled design of 
Lemma 4.3 may exhibit unacceptable transient behaviour in response to step inputs due to cross-coupling in the closed-loop transfer function. For the diagonally decoupled system of Lemma 4.6, the cost of decoupling, manifested in the diagonal matrix $D$ of Definition 4.2, may produce unacceptable overshoots due to the non-minimum phase elements of $D$. Moreover, these effects are to a large extent unaffected by the design variables $a_{1}, \cdots, a_{p}, a_{\mathrm{n}}$ in the generalized interactor matrix.

By introducing one additional scalar design variable, it is possible to blend the designs for partial and diagonal decoupling in a manner that permits a trade-off between transient cross-coupling and the additional non-minimum phase zeros introduced as the cost of diagonal decoupling. A key observation is that the choice

$$
Q=K^{-1}
$$

leads to the partially decoupled design of Lemma 4.3, while

$$
Q=K^{-1} \Sigma D
$$

provides diagonal decoupling. It is possible to unify these designs by selecting

$$
Q=\lambda\left(K^{-1} \Sigma D\right)+(1-\lambda) K^{-1}, \quad 0 \leq \lambda \leq 1 .
$$

The key properties of this design are summarized in the following Theorem, which is the main result of the paper.

Theorem 5.1 Let $P(s) \in \mathbf{R}_{\mathrm{sp}}^{m \times m}(s)$ be stable and nonsingular, let $\xi(s)=$ $\Sigma(s) \Delta(s)$ be the generalized interactor matrix of $P(s)$, and let $D(s)$ be as in Definition 4.2. Then the design

$$
Q=\lambda\left(K^{-1} \Sigma D\right)+(1-\lambda) K^{-1}, \quad 0 \leq \lambda \leq 1
$$

achieves static and partial decoupling (and hence internal stability) of $S(P, C)$ such that the closed-loop transfer function is given by

$$
H_{y r}=\Delta^{-1} \Sigma^{-1}[\lambda(\Sigma D)+(1-\lambda) I], \quad 0 \leq \lambda \leq 1 .
$$

Moreover, $\lambda=0$ yields the partially decoupled design of Lemma 4.3, and $\lambda=1$ yields the diagonally decoupled design of Lemma 4.6.

Proof. From Lemma 4.2 and Definition 4.2 , both $K^{-1}$ and $K^{-1} \Sigma D$ are proper and stable, thus $\lambda\left(K^{-1} \Sigma D\right)$ and $(1-\lambda) K^{-1}$ are proper and stable for $\lambda \in[0,1]$. Since the set of matrices of proper and stable transfer functions forms a ring, it is closed under addition, therefore $Q$ given by (28) is proper 
and stable, and $S(P, C)$ is internally stable. The closed-loop transfer function $H_{y r}$ is given by

$$
\begin{aligned}
H_{y r} & =P Q \\
& =\xi^{-1} K\left[\lambda\left(K^{-1} \Sigma D\right)+(1-\lambda) K^{-1}\right] \\
& =(\Sigma \Delta)^{-1}[\lambda(\Sigma D)+(1-\lambda) I]
\end{aligned}
$$

as given in (29). The closed-loop system $S(P, C)$ is statically decoupled since

$$
\begin{aligned}
H_{y r}(0) & =\Delta^{-1}(0) \Sigma^{-1}(0)[\lambda(\Sigma(0) \Delta(0))+(1-\lambda) I] \\
& =I[\lambda I+(1-\lambda) I] \\
& =I
\end{aligned}
$$

and partially decoupled since from $(29),(\Sigma \Delta)^{-1}$ is lower triangular while $\lambda(\Sigma D)$ and $(1-\lambda) I$ are diagonal. If $\lambda=0$,

$$
H_{y r}=\Delta^{-1} \Sigma^{-1}=\xi^{-1}
$$

as in Lemma 4.3 , and if $\lambda=1$,

$$
H_{y r}=\Delta^{-1} \Sigma^{-1} \Sigma D=\Delta^{-1} D
$$

as in Lemma 4.6.

Thus the design (28) leads to a family of partially decoupled closed-loop transfer matrices in which diagonal decoupling arises as a special case, namely when $\lambda=1$. It should be noted that this family is by no means all partially decoupled closed-loop transfer matrices. Indeed, it follows from the proof of Lemma 5.1 that any value of $\lambda$ (not necessarily in $[0,1]$ ) results in an internally stable closed-loop system. Nevertheless, closed-loop systems arising from the use of $\lambda \notin[0,1]$ fail to exhibit the desirable properties of either the partially decoupled or diagonally decoupled designs (25) and (26) respectively.

\section{Design example}

Consider a 2-input 2-output plant whose transfer matrix is given by

$$
P=\left[\begin{array}{cc}
\frac{(1-s)(s+2)}{(2 s+1)(s+1)(s+4)} & \frac{(1-s)(s+2)}{(2 s+1)(s+1)(s+3)} \\
\frac{-\left(s^{4}+7 s^{3}+14 s^{2}+6 s-4\right)}{(2 s+1)^{2}(s+1)(s+3)(s+4)} & \frac{-(s+2)\left(s^{3}+4 s^{2}+3 s+4\right)}{(2 s+1)^{2}(s+1)(s+3)(s+5)}
\end{array}\right]
$$


which is stable, nonsingular, strictly proper, has a single right half-plane zero at $s=1$ of multiplicity two, and no unstable hidden modes. The generalized interactor satisfies

$$
\xi P=K
$$

where from Definition 4.2,

$$
\begin{aligned}
\xi & =\xi_{z_{1}} \xi_{\mathrm{n}}, \\
K & =K_{2}=\xi_{z_{1}} K_{1}, \\
K_{1} & =\xi_{\mathrm{n}} P .
\end{aligned}
$$

Computing the normalized interactor $\xi_{\mathrm{n}}$ of $P$ using the method of Wolovich and Falb (Wolovich and Falb 1976) and normalizing as described in Lemma 3.1 with $a_{\mathrm{n}}=2$, we find

$$
\begin{aligned}
\xi_{\mathrm{n}} & =\Sigma_{\mathrm{n}} \Delta_{\mathrm{n}} \\
& =\left[\begin{array}{cc}
1 & 0 \\
-s & 1
\end{array}\right]\left[\begin{array}{cc}
2 s+1 & 0 \\
0 & (2 s+1)^{2}
\end{array}\right] \\
& =\left[\begin{array}{cc}
2 s+1 & 0 \\
-s(2 s+1) & (2 s+1)^{2}
\end{array}\right],
\end{aligned}
$$

and

$$
K_{1}(s)=\left[\begin{array}{cc}
\frac{(1-s)(s+2)}{(s+1)(s+4)} & \frac{(1-s)(s+2)}{(s+1)(s+3)} \\
\frac{-\left(3 s^{3}+13 s^{2}+12 s-4\right)}{(s+1)(s+3)(s+4)} & \frac{-4(s+2)(2 s+1)}{(s+1)(s+3)(s+5)}
\end{array}\right] .
$$

The next step is to extract the $z$-interactor of $K_{1}$ as in Eq. (32) for $z_{1}=1$. Using the three step procedure following Lemma 4.1:

Step 1. Since $z_{1}=1$, choose $a_{1}=1 / z_{1}=1$ as per (24). Thus,

$$
\begin{aligned}
K_{1}\left(s^{\prime}\right) & =\left.K_{1}(s)\right|_{s=\frac{s^{\prime}}{s^{\prime}+2}} \\
& =\left[\begin{array}{cc}
\frac{3 s^{\prime}+4}{\left(5 s^{\prime}+8\right)\left(s^{\prime}+1\right)} & \frac{3 s^{\prime}+4}{2\left(2 s^{\prime}+3\right)\left(s^{\prime}+1\right)} \\
\frac{-\left(12 s^{\prime 3}+25 s^{\prime 2}-16\right)}{2\left(5 s^{\prime}+8\right)\left(2 s^{\prime}+3\right)\left(s^{\prime}+1\right)} & \frac{-\left(s^{\prime}+2\right)\left(3 s^{\prime}+2\right)\left(3 s^{\prime}+4\right)}{2\left(3 s^{\prime}+5\right)\left(2 s^{\prime}+3\right)\left(s^{\prime}+1\right)}
\end{array}\right] .
\end{aligned}
$$

Step 2. Compute the normalized interactor of $K_{1}\left(s^{\prime}\right)$ :

$$
\xi_{z_{1}}(s)=\left[\begin{array}{cc}
s^{\prime}+1 & 0 \\
s^{\prime}\left(s^{\prime}+1\right) & s^{\prime}+1
\end{array}\right] .
$$


Step 3. Obtain $\xi_{z_{1}}(s)$ :

$$
\begin{aligned}
\xi_{z_{1}}(s) & =\left.\xi_{z_{1}}\left(s^{\prime}\right)\right|_{s^{\prime}=\frac{-2 s}{s-1}} \\
& =\left[\begin{array}{cc}
-\left(\frac{s+1}{s-1}\right) & 0 \\
\frac{2 s(s+1)}{(s-1)^{2}} & -\left(\frac{s+1}{s-1}\right)
\end{array}\right] .
\end{aligned}
$$

Then from Eqs. (31), (34) and (35),

$$
\begin{aligned}
\xi & =\left[\begin{array}{cc}
\frac{-(s+1)(2 s+1)}{s-1} & 0 \\
\frac{s(s+1)^{2}(2 s+1)}{(s-1)^{2}} & \frac{-(s+1)(2 s+1)^{2}}{s-1}
\end{array}\right] \\
& =\Sigma \Delta,
\end{aligned}
$$

where

$$
\begin{aligned}
& \Sigma=\left[\begin{array}{cc}
1 & 0 \\
\frac{-s(s+1)}{s-1} & 1
\end{array}\right], \\
& \Delta=\left[\begin{array}{cc}
\frac{-(2 s+1)(s+1)}{s-1} & 0 \\
0 & \frac{-(s+1)(2 s+1)^{2}}{s-1}
\end{array}\right],
\end{aligned}
$$

and

$$
\begin{aligned}
K & =\xi P \\
& =\left[\begin{array}{cc}
\frac{s+2}{s+4} & \frac{s+2}{s+3} \\
\frac{(s+2)^{2}}{(s+3)(s+4)} & \frac{-2(s+2)^{2}}{(s+3)(s+5)}
\end{array}\right] .
\end{aligned}
$$

Clearly $\Sigma$ is unstable and improper. Using Definition 4.2 to identify $D$ such that $\Sigma D$ is proper and stable:

$$
D=\left[\begin{array}{cc}
\frac{1-s}{(s+1)(2 s+1)} & 0 \\
0 & 1
\end{array}\right]
$$

From Theorem 5.1, a family of partially decoupled closed-loop transfer functions is given by

$$
H_{y r}=\left[\begin{array}{cc}
\frac{(s-1)\left(s^{2}[2 \lambda-2]+s[2 \lambda-3]-1\right)}{(2 s+1)^{2}(s+1)^{2}} & 0 \\
\frac{s(\lambda-1)}{(2 s+1)^{2}} & \frac{1-s}{(2 s+1)^{2}(s+1)}
\end{array}\right]
$$


The choice $\lambda=0$ yields the following partially decoupled transfer matrix:

$$
\begin{aligned}
H_{y r} & =\xi^{-1} \\
& =\left[\begin{array}{cc}
\frac{1-s}{(2 s+1)(s+1)} & 0 \\
\frac{-s}{(2 s+1)^{2}} & \frac{1-s}{(2 s+1)^{2}(s+1)}
\end{array}\right],
\end{aligned}
$$

while $\lambda=1$ yields the following diagonally decoupled transfer matrix:

$$
\begin{aligned}
H_{y r} & =\Delta^{-1} D \\
& =\left[\begin{array}{cc}
\frac{(s-1)^{2}}{(2 s+1)^{2}(s+1)^{2}} & 0 \\
0 & \frac{1-s}{(2 s+1)^{2}(s+1)}
\end{array}\right] .
\end{aligned}
$$

The closed-loop performance of the system, as described by Eq. (36), has been simulated for $\lambda=0,0.5$, and 1 , and the results are presented in Figure 2. Overlaying the plots enables the effect of $\lambda$ on the closed-loop performance to be readily assessed.

With $\lambda=0$ (indicated by dotted lines $\cdots$ ), the first plant output $y_{1}$ responds rapidly to step changes in the reference input $r_{1}$, but induces a significant transient into the second output $y_{2}$. By increasing $\lambda$ to 0.5 (indicated by dashdot lines $-\cdot-$ ), the peak magnitude of this transient is reduced by a factor of one half at the expense of increased rise-time of $y_{1}$ to step changes in $r_{1}$. Increasing $\lambda$ to 1 (indicated by dashed lines --) produces a diagonally decoupled closed-loop transfer function, as evidenced by the absence of any transient in $y_{2}$ due to step changes in $r_{1}$, but the response time of $y_{1}$ is increased yet again. This behaviour clearly demonstrates the cost of decoupling. Diagonal decoupling is achieved at the expense of introducing additional non-minimum phase zeros and increased relative degree (zeros at $s=\infty)$ into the closed-loop transfer function; cf. the $(1,1)$ entries of the transfer matrices in Eqs. (37) and (38).

Note also that for $y_{2}$ there is no cost of decoupling in terms of these additional zeros. The response time of $y_{2}$ to step changes in $r_{2}$ is independent of the value of $\lambda$. This is true in general for the $m$ th output of an $m$-input $m$-output system since the $m$ th column of $\Sigma=[0, \cdots, 0,1]^{T}$ so that from Definition $4.2, D_{m} \equiv 1$.

\section{Conclusions}

In this paper a controller design method which achieves partial closed-loop decoupling for stable, linear time-invariant square MIMO plants has been 

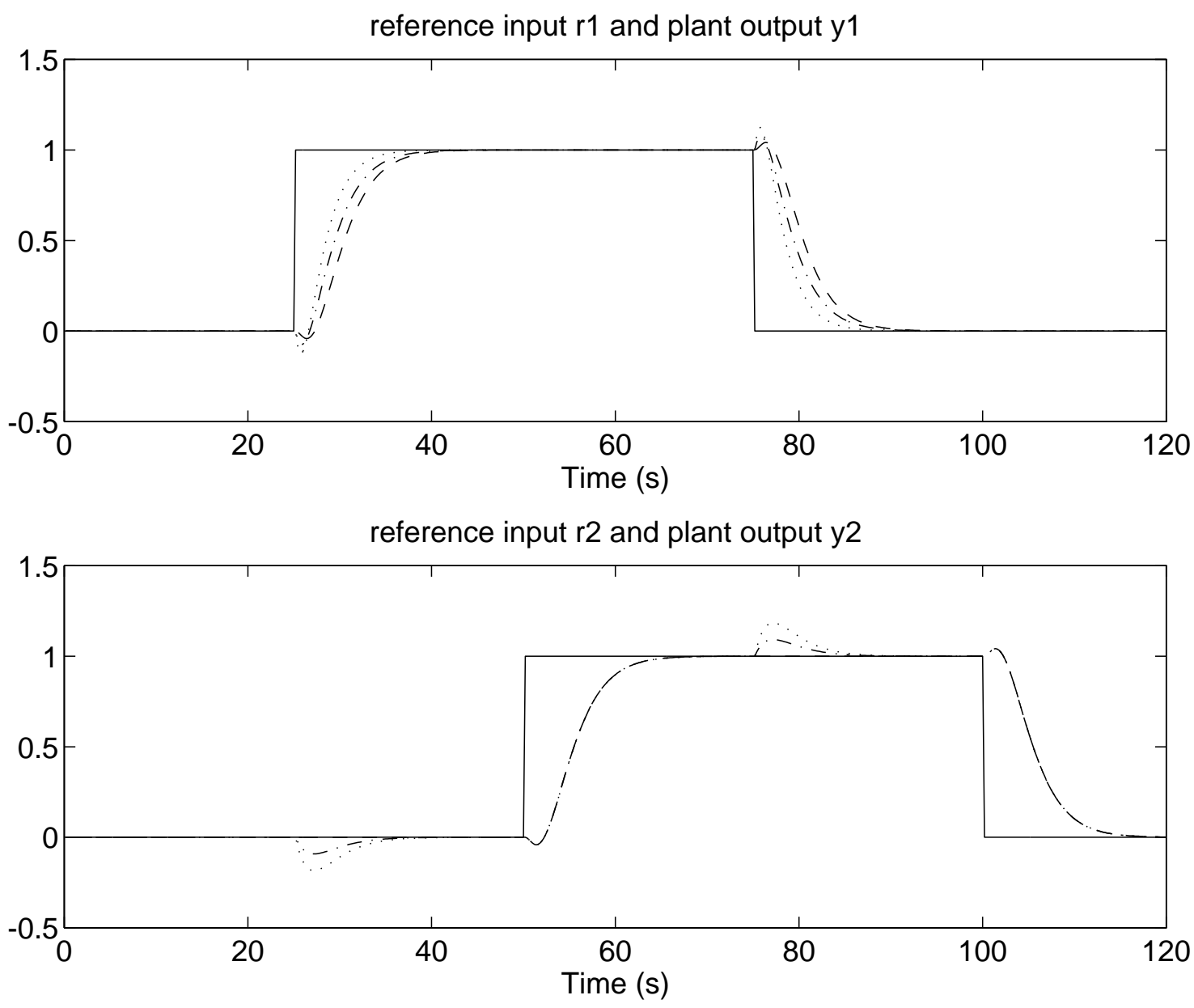

Figure 2: Closed-loop behaviour of example system for $\lambda=0(\cdots), \lambda=0.5(-$. $-)$ and $\lambda=1(--)$. As $\lambda$ is increased from 0 to 1 , the size of the transient induced in plant output $y_{2}$ as a consequence of a change in reference input $r_{1}$ decreases at the expense of increased rise-time in $y_{1}$ 
presented. The method utilizes a factorization of the plant transfer matrix provided by the generalized interactor, which contains a representation of the non-minimum phase zeros and the zero structure at infinity of the plant. In the proposed method, a scalar tuning variable permits the speed of closedloop response to be traded-off against transient interactions between loops, and diagonal decoupling is achieved as a special case of partial decoupling.

\section{References}

Callier, F. and Desoer, C., 1982, Multivariable Feedback Systems, SpringerVerlag.

Chang, F.-R. and Wang, L.-C., 1990, Algorithms for the interactor matrix and the row-column-reduced form, Control-Theory and Advanced Technology 6(3), 483-491.

Desoer, C. and Gündeş, A., 1986, Decoupling linear multiinput multioutput plants by dynamic output feedback: An algebraic theory, IEEE Trans. Automat. Control AC-31(8), 744-750.

Desoer, C., Liu, R.-W., Murray, J. and Saeks, R., 1980, Feedback system design: The fractional representation approach to analysis and synthesis, IEEE Trans. Automat. Control AC-25(3), 399-412.

Dugard, L., Goodwin, G. and Xianya, X., 1984, The role of the interactor matrix in multivariable stochastic adaptive control, Automatica 20(5), 701709.

Elliott, H. and Wolovich, W., 1982, A parameter adaptive control structure for linear multivariable systems, IEEE Trans. Automat. Control AC27(2), 340-352.

Furuta, K. and Kamiyama, S., 1977, State feedback and inverse system, Int. J. Control 25(2), 229-241.

Hung, N. and Anderson, B., 1979, Triangularization technique for the design of multivariable control systems, IEEE Trans. Automat. Control AC24(3), 455-460.

Middleton, R. and Goodwin, G., 1990, Digital Control and Estimation: A Unified Approach, Prentice-Hall, Englewood Cliffs, NJ. 
Morari, M. and Zafiriou, E., 1989, Robust Process Control, Prentice-Hall, Englewood Cliffs, NJ.

Pernebo, L., 1981, An algebraic theory for the design of controllers for linear multivariable systems - Part I: Structure matrices and feedforward design, IEEE Trans. Automat. Control AC-26(1), 171-182.

Skogestad, S., Morari, M. and Doyle, J., 1988, Robust control of illconditioned plants: High-purity distillation, IEEE Trans. Automat. Control 33(12), 1092-1105.

Stephanopoulos, G., 1984, Chemical Process Control: An Introduction to Theory and Practice, Prentice-Hall, Englewood Cliffs, NJ.

Tsiligiannis, C. and Svoronos, S., 1989, Dynamic interactors in multivariable process control-II: Time delays and zeroes outside the unit circle, Chem. Eng. Sci. 44(9), 2041-2047.

Vardulakis, A., 1991, Linear Multivariable Control: Algebraic Analysis and Synthesis Methods, John Wiley \& Sons Ltd., Chichester, England.

Vardulakis, A. and Karcanias, N., 1982, On the stable exact model matching problem, Systems \& Control Letters 5(4), 237-242.

Weller, S., 1993, Structural issues in control design for linear multivariable systems, PhD thesis, University of Newcastle, Newcastle, New South Wales, Australia.

Weller, S. and Goodwin, G., 1995, Partial decoupling of unstable linear multivariable systems, Proc. 3rd European Control Conf., Rome, Italy, pp. 2539-2544.

Wolovich, W. and Falb, P., 1976, Invariants and canonical forms under dynamic compensation, SIAM J. Control and Optimization 14(6), 9961008.

Youla, D., Jabr, H. and Bongiorno, J., 1976, Modern Wiener-Hopf design of optimal controllers - Part II: The multivariable case, IEEE Trans. Automat. Control AC-21(6), 319-338.

Zafiriou, E. and Morari, M., 1987, Digital controller design for multivariable systems with structural closed-loop performance specifications, Int. J. Control 46(6), 2087-2111. 
Zames, G., 1981, Feedback and optimal sensitivity: Model reference transformations, multiplicative seminorms and approximate inverses, IEEE Trans. Automat. Control AC-26(2), 301-320. 